\title{
Characterization of a Surface Micromachined Pressure Sensor Array
}

\author{
W.P. Eaton and J.H. Smith \\ Integrated Micromechanics, Microsensors, \& CMOS Technologies Department \\ Sandia National Laboratories \\ Albuquerque, NM 87185-1080
}

Keywords: surface micromachining, MEMS, CMOS, pressure sensor, pressure transducer, vacuum encapsulated

\begin{abstract}
A surface micromachined pressure sensor array has been designed and fabricated. The sensors are based upon deformable, silicon nitride diaphragms with polysilicon piezoresistors. Absolute pressure is detected by virtue of reference pressure cavities underneath the diaphragms. For this type of sensor, design tradeoffs must be made among allowable diaphragm deflection, diaphragm size, and desirable pressure ranges. Several fabrication issues were observed and addressed. Offset voltage, sensitivity, and nonlinearity of $100 \mu \mathrm{m}$ diameter sensors were measured.
\end{abstract}

\section{INTRODUCTION}

Recently, there has been a great deal of interest in micromachined sensors and actuators. The Microelectronics Development Laboratory (MDL) at Sandia National Laboratories has developed a surface micromachined pressure sensor which complements other projects and is intended to be used as a modular building block for microsystems applications. In addition, there are other specific industrial and defense applications for this sensor. All materials and processes used for sensor fabrication are CMOS compatible, and are part of the ongoing effort of CMOS integration with MEMS at the MDL ${ }^{1}$.

While the principal objective of the project was to develop a robust, wide-range pressure sensor technology, many specific goals were put forth. The required pressure range of the sensor array is 1-10 atm (absolute), with frequency responses from DC to $2 \mathrm{MHz}$. Most of the processing equipment used for micromechanics at the MDL is shared with a $0.5 \mu \mathrm{m}$ CMOS line. The requirement for $100 \%$ CMOS compatibility was to guarantee that sensor lots would not contaminate equipment, and also to allow integration with CMOS circuitry if necessary. Conceivable electronic functions are: signal conditioning, calibration, temperature compensation, analog to digital conversion, or network communication protocol. Finally, the desired operating environments were gaseous (including air), liquid, and severe (e.g. corrosive). Operation in all of these media provides enhanced flexibility of applications.

\section{EXPERIMENTAL}

\subsection{Sensor Design}

The two principal design considerations for a diaphragm-based pressure sensor are diaphragm geometry and piezoresistor size and placement. These piezoresistors act as strain gauges that change their resistance value according to the deflected state of the diaphragm. This sensor design utilizes both circumferentially and radially oriented piezoresistors, which have opposite resistance changes. This feature can be used to increase the overall sensitivity of the device. Details of piezoresistor size and placement are described elsewhere ${ }^{2,3}$

The geometry of the diaphragm effects the mechanical deflection under applied pressure and, hence, the sensitivity and range of the sensor. A circular geometry was chosen for the pressure sensor. Circular symmetry lends itself to analytical solutions of plate theory. The deflection at the center of an edge-clamped plate due to a uniform applied pressure is given by Timoshenko ${ }^{4}$ as:

- Under contract from the Center for High Technology Materials, The University of New Mexico, Albuquerque, NM 87131. 


\section{DISCLAIMER}

Portions of this document may be illegible in electronic image products. Images are produced from the best available original document. 


$$
w=\frac{P a^{4}}{64 D}, \quad D=\frac{E h^{3}}{12\left(1-v^{2}\right)}
$$

and the corresponding radial and circumferential strains are:

$$
\begin{aligned}
& \varepsilon_{r}=-\frac{3 P a^{2}\left(1-v^{2}\right)}{8 E h^{2}}\left[1-3\left(\frac{r}{a}\right)^{2}\right] \\
& \varepsilon_{\theta}=-\frac{3 P a^{2}\left(1-v^{2}\right)}{8 E h^{2}}\left[1-\left(\frac{r}{a}\right)^{2}\right]
\end{aligned}
$$

where

$$
\begin{array}{ll}
a \equiv \text { diaphragm radius [m] } \quad D \equiv \text { flexural rigidity }[\mathrm{N} \cdot \mathrm{m}] \text { or [Joule] } & \left.E \equiv \text { Young's modulus [N/ } \mathrm{m}^{2}\right] \text { or }[\mathrm{Pa}] \\
\varepsilon_{r}, \varepsilon_{0} \equiv \text { radial, circumferential strain [unitless] } h \equiv \text { diaphragm thickness [m] } & v \equiv \text { Poisson's ratio [unitless] } \\
P \equiv \text { applied pressure }\left[\mathrm{N} / \mathrm{m}^{2} \text { ] or }[\mathrm{Pa} \quad \quad w \equiv \text { diaphragm deflection }[\mathrm{m}]\right. & r \equiv \text { radial coordinate [m] (zero at center) }
\end{array}
$$

Materials constants for silicon nitride are given in the caption of Table 1. Equations (1) and (2) describe deflections that are proportional to $\left(a^{4} / h^{3}\right)$, but strains (and piezoresistive sensitivities) that are proportional to $\left(a^{2} / h^{2}\right)$. For the pressure sensor presented here, the allowed deflection is controlled by the thickness of the sacrificial oxide stack. Thus, design tradeoffs must be made among allowable deflection, diaphragm size, and desirable pressure ranges. A thorough description of a design process of a similar sensor is presented by Burns 3

Seven sizes of diaphragms were designed. Resonant frequencies and maximum applied pressure before full deflection were approximated by using simple plate theory and are shown in Table 1. By using an array of diaphragm sizes, a wide range of pressures can be accurately sensed. Table 1 suggests that the larger sensors should be fully deflected at atmospheric pressure. These sensors may find use as vacuum sensors and in other low pressure applications. Overpressure bursting of large membranes while operating in the pressure regime of the small membranes is not expected to be an issue since the diaphragm travel will be stopped by the underlying substrate. Excellent overpressure behavior of similar surface micromachined pressure sensors has been demonstrated by other researchers 5 .

\begin{tabular}{|l|r|c|l|}
\hline \multicolumn{1}{|l}{$\mathbf{h}[\boldsymbol{m}]$} & \multicolumn{1}{c}{$\mathbf{a}[\boldsymbol{\mu m}]$} & $f_{0}[\mathrm{MHz}]$ & $\mathbf{P}_{\max }$ [psia $]$ \\
\hline 0.8 & 25 & 7.0 & 650 \\
\hline 0.8 & 50 & 1.8 & 41 \\
\hline 0.8 & 75 & 0.78 & 8.0 \\
\hline 0.8 & 100 & 0.44 & 2.5 \\
\hline 0.8 & 125 & 0.28 & 1.0 \\
\hline 0.8 & 250 & 0.070 & 0.06 \\
\hline 0.8 & 500 & 0.018 & 0.004 \\
\hline
\end{tabular}

Table 1. Theoretical diaphragm characteristics with varied diaphragm radius. Materials constants for $\mathrm{Si}_{3} \mathrm{~N}_{4}$ :

$E=300[\mathrm{GPa}], \rho=2.3\left[\mathrm{~g} / \mathrm{m}^{3}\right], v=.25$

A schematic representation of the pressure sensor is shown in Figure 1. A circular silicon nitride diaphragm forms the top of a sealed vacuum cavity, which provides a reference pressure. Polysilicon strain gauges are mounted on top of the diaphragm. As the membrane deflects due to an applied pressure, the edges of the diaphragm are placed in radial tensile strain, while the center is placed in circumferential compressive strain. For properly designed resistors, this behavior causes resistance changes in the radial and circumferential strain gauges that are equal but opposite in sign. To first order, the resistance changes are directly proportional to the applied pressure. 


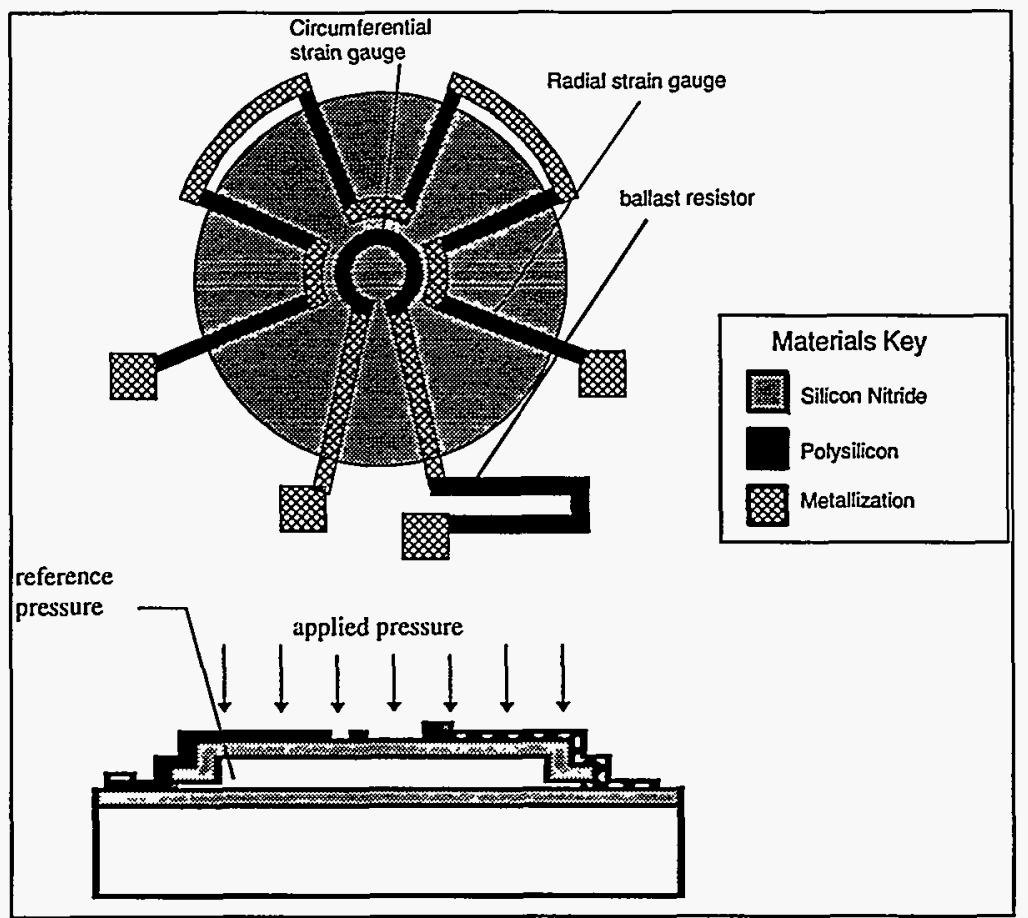

Figure 1. Schematic of pressure sensor.

\subsection{Fabrication Sequence}

The fabrication sequence of the sensor is shown schematically in Figure 2 and in optical and electron micrographs in Figure 3. The first step is to deposit a $0.8 \mu \mathrm{m}$ thick base layer of low stress silicon nitride film (Figure 2a). This acts as a insulating base layer for resistor and metal lines, and as a non-stick layer so that a fully deflected membrane is less likely to adhere to the substrate. The nitride is deposited in a VTR vertical low pressure chemical vapor deposition (LPCVD) furnace and the resulting film thicknesses generally vary from the top to the bottom of the boat and radially accross a single wafer. This is due to diffusion limitations during deposition. Thickness variations are not an issue for the base layer, but become more important for the diaphragm layer.

Following the base layer nitride deposition an LPCVD oxide based on tetra-ethyl-orthosilicate (TEOS) is deposited. The deposition is $2 \mu \mathrm{m}$ thick and is performed in a VTR vertical tube furnace. The resultant film is patterned as shown in Figure 2(b) and Figure 3(a). Then, a thinner 0.1 $\mu \mathrm{m}$ layer of LPCVD boro-phospho-silicate glass (BPSG) is deposited and patterned (Figure 2(c)). This glass is also TEOS based, but is doped with 5\% boron and 5\% phosphorous and is deposited in a LAM Integrity reactor. The combined thickness of the thin and thick oxides determine the maximum diaphragm deflection. The composite film of TEOS and BPSG is used to achieve a faster release etch rate than would be attainable by TEOS alone.

After the oxide deposition steps, another $0.8 \mu \mathrm{m}$ film of low-stress silicon nitride is deposited and patterned (Figure $2 \mathrm{~d}$ ). This layer of nitride is the diaphragm material and is anchored to the base layer of nitride through cuts in the sacrificial oxide layers. During the dry etch of the nitride, the sidewalls of the photoresist are preferentially etched. This causes failure of the photoresist and local thinning of the nitride. This problem can be addressed by depositing a $0.3-0.5 \mu \mathrm{m}$ thick layer of TEOS as a hard mask before the photolithography. The TEOS can be etched in the same chamber as the nitride, and is removed during the release etch.

Figure 3(b) shows a nitride diaphragm after deposition but before patterning. After patterning, thin oxide stubs are exposed. These thin oxide stubs allow etchant to reach the oxide layers underneath the diaphragm during the release etch. The devices are placed in an HF-based, release etch solution. This solution removes the oxide layers beneath the diaphragm, thus freeing the diaphragm. The thin oxide of BPSG etches much faster than the TEOS layer. During the release etch, the BPSG etches rapidly in the lateral direction, exposing the thick oxide. The exposed thick oxide must only be etched through its thickness and is etched quickly. Hence, the net lateral etch rate of the thin/thick oxide stack approaches that of the BPSG. 
Figure 3(c) shows a partially-released $100 \mu \mathrm{m}$-diameter diaphragm. The white material in the center is unetched oxide beneath the nitride diaphragm. A fully-released 50 $\mathrm{m}$ diaphragm is shown in Figure 3(d), with the inset showing a close-up of one of the etch ports. The etch port was attacked by the HF during the release etch. This nitride attack can be lessened by using concentrated solutions of $\mathrm{HF}$ or concentrated solutions of $\mathrm{HF}: \mathrm{HCl}^{6.7}$. After the oxide has been etched away in the $\mathrm{HF}$ solution, the wafers are dilution rinsed with deionized water, removed from the water, and allowed to air dry.

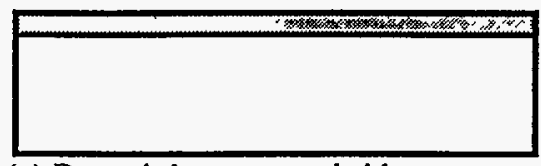

(a) Deposit low stress nitride.

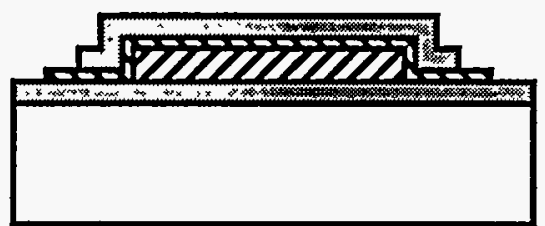

(d) Deposit and pattern diaphragm nitride.

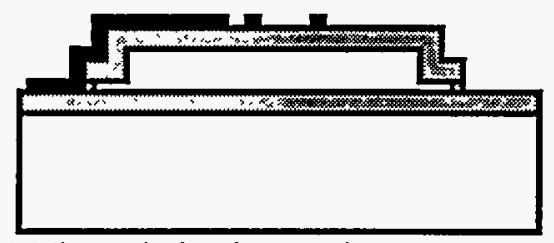

(g) Deposit, implant, and pattern piezoresistive polysilicon

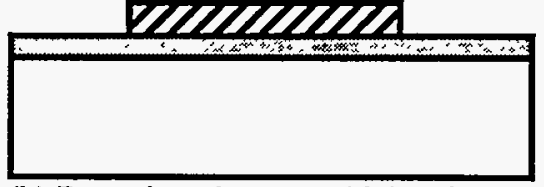

(b) Deposit and pattern thick oxide.

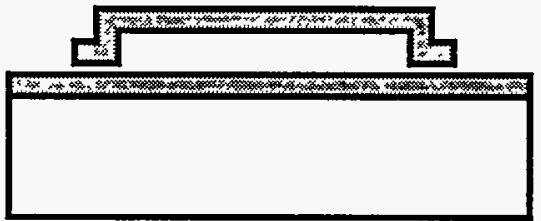

(e) Etch oxides in HF solution.

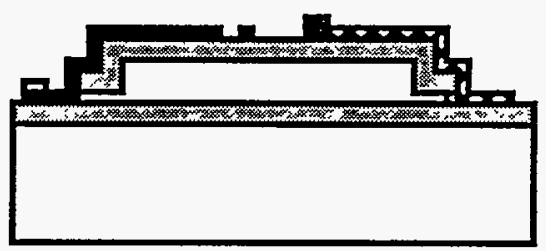

(h) Deposit and pattern aluminum.

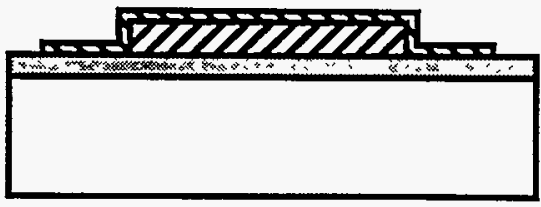

(c) Deposit and pattern thin oxide.

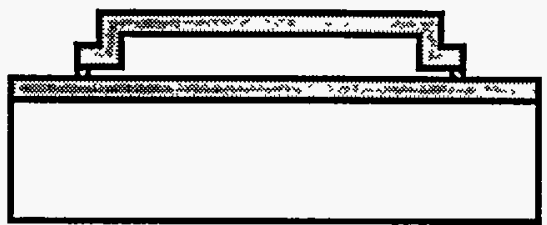

(f) Seal diaphragms with nitride.

Figure 2. Schematic fabrication sequence of microsensors.

Following the drying process, the diaphragms are sealed by another low-stress nitride deposition of $\sim 0.1 \mu \mathrm{m}$, which plugs the etch ports. If the etch ports are significantly enlarged during the HF etch, the sealing layer thickness must be increased. Ideally, without the attack of the nitride during the release etch, the minimum thickness of the sealing layer would be half the thickness of the thin oxide. Deposition conditions during the sealing step are $850^{\circ} \mathrm{C}$ and $250 \mathrm{mTorr}$, so the residual pressure at room temperature (assuming ideal gases) should be $\approx 67 \mathrm{mTorr}$. Some researchers have reported residual pressures of 200-300 mTorr ${ }^{8}$ for nitride sealing. The actual pressure, though stable for any given diaphragm, may be variable and non-repeatable across a substrate and from substrate to substrate. However, since residual pressures are under 1 Torr, the pressure variations are insignificant relative to the sensitivity of the sensors for pressure measurements at or above atmospheric pressure. Other sealing techniques can be used such as polysilicon deposition ${ }^{8}$ or reactive oxide sealing ${ }^{3}$.

Once the diaphragms have been sealed, special rinsing and drying techniques are no longer needed. Processing continues with the deposition and ion implantation of the polysilicon piezoresistors. An implant of phosphorus at $80 \mathrm{keV}$ with a dose of $3 \cdot 10^{14} / \mathrm{cm}^{2}$ was chosen to maximize the gauge factor of the polysilicon ${ }^{9}$. After implant, the polysilicon is patterned. Finally, aluminum metallization is deposited and patterned, and the devices are ready to be tested. For corrosion resistance, an additional silicon nitride layer can be deposited and patterned as a passivation layer.

Not shown in Figure 2 is a special shunt layer of in situ doped polysilicon. This layer ameliorates the step coverage problems of the aluminum over the diaphragm edges. The shunt layer is patterned with the same mask as the aluminum layer, but is placed directly underneath the piezoresistors. In planar regions, current will flow preferentially through the low resistance path of the aluminum. Where there are breaks in aluminum over steps, current will flow through the poly shunt 
layer. The shunt layer can be avoided altogether if more planar topologies are used, such as in references 3 and 10. A planar topology is currently under investigation.
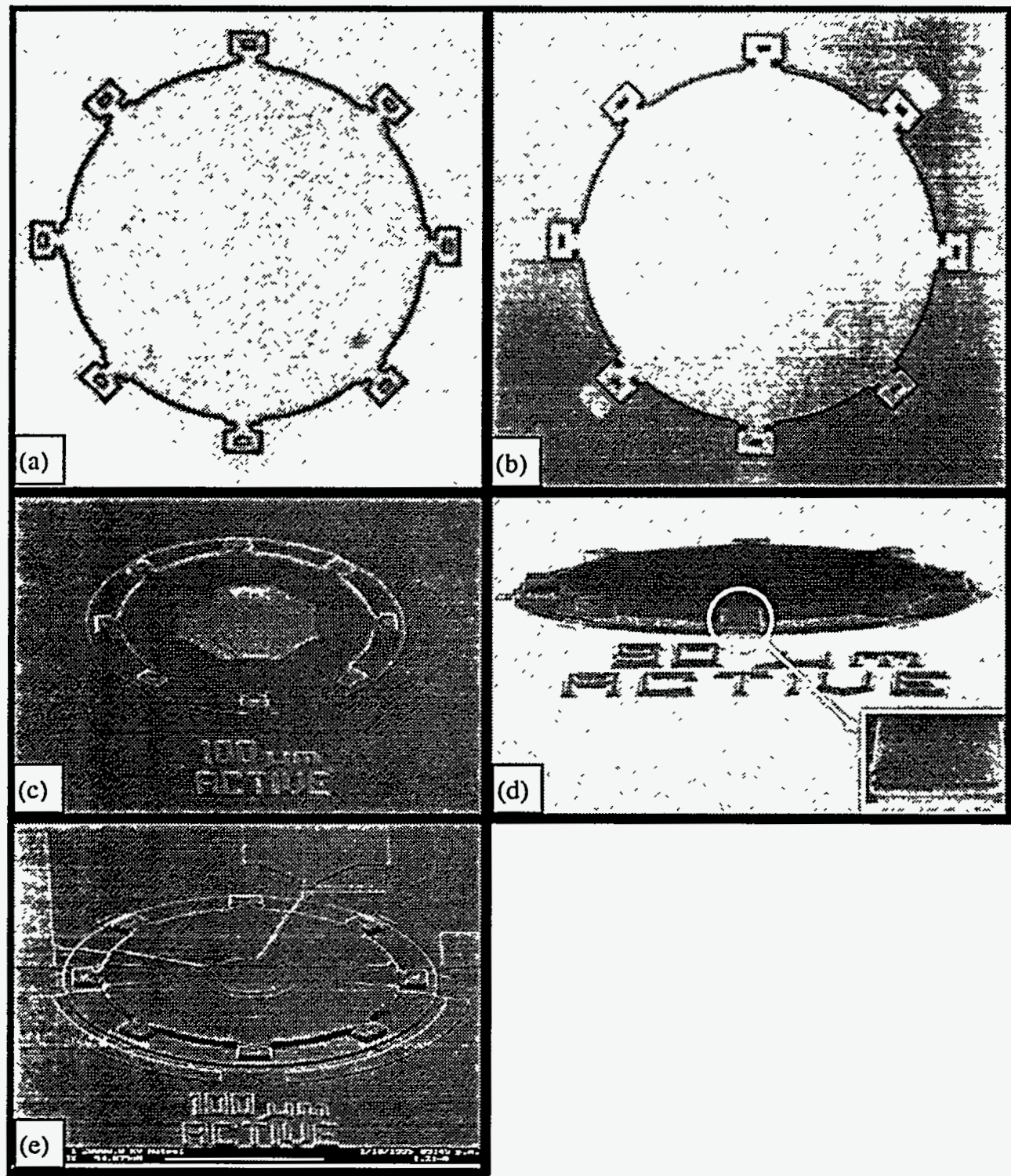

Figure 3. Optical[(a),(b)] and electron[(c)-(e)] micrographs of fabrication sequence. (a) $100 \mu \mathrm{m}$ diameter thıck oxide after patternıng (b) $100 \mu \mathrm{m}$ diameter diaphragm after diaphragm deposition. (c) Partially released $100 \mu \mathrm{m}$ diameter diaphragm. (d) Fully released $50 \mu \mathrm{m}$ diameter diaphragm. (e) Completed I0Opm dianeter pressure sensor.

\subsection{Signal detection}

Signal detection is accomplished by connecting the piezoresistive strain gauges in a Wheatstone bridge configuration. This approach has several advantages: 1) first order compensation of common mode signals such as temperature 2) voltage output, which is easily measured, and 3) relative immunity to changes in supply voltage. A current loop detection scheme has similar advantages ${ }^{11,12}$, but has slightly more complicated instrumentation requirements.

Three bridge configurations were used: fully active, half active circumferential, and half active radial. These configurations are shown in Figure 4. $R_{u c}, R_{d c}, R_{u r}$, and $R_{d r}$ are active and dummy circumferential resistors and active and dummy radial resistors, respectively. Active resistors are placed upon diaphragms and are sensitive to applied pressure, whereas dummy resistor's are placed either on unreleased diaphragms or in the field region and are insensittve to applied pressure. Following the schematics of Figure 1 and Figure 4, two diaphragms are required for one fully active bridge sensor, 

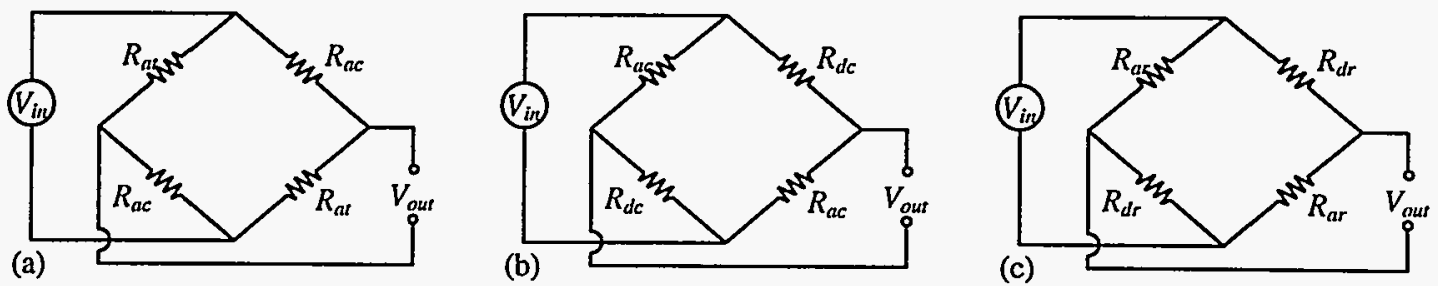

Figure 4. Wheatstone bridge configurations: (a) fully active, (b) half active circumferential, and (c) half active radial.

but four diaphragms ( 2 active and 2 dummy) are required for one half active bridge sensor. If well matched resistances, $R$, and resistance changes, $\Delta R$, are assumed then the circumferential and radial resistors will have total resistances under applied pressure of

$$
\begin{array}{ll}
R_{a c}=\Delta R+R, & R_{d c}=R \\
R_{u r}=\Delta R-R, & R_{d r}=R
\end{array}
$$

The output voltages become

$$
\left.\begin{array}{lll}
\text { fully active: } & V_{\text {out }}=\left(\frac{R+\Delta R}{2 R}-\frac{R-\Delta R}{2 R}\right) V_{\text {in }}=\frac{\Delta R}{R} V_{\text {in }} \\
\text { half active circumferential: } V_{\text {out }}=\left(\frac{R}{2 R+\Delta R}-\frac{R+\Delta R}{2 R+\Delta R}\right) V_{\text {in }}=\left(\frac{-\Delta R}{2 R+\Delta R}\right) V_{\text {in }} \approx \frac{-\Delta R}{2 R} V_{\text {in }} \\
\text { half active radial: } & V_{\text {out }}=\left(\frac{R}{2 R-\Delta R}-\frac{R-\Delta R}{2 R-\Delta R}\right) V_{\text {in }}=\left(\frac{\Delta R}{2 R+\Delta R}\right) V_{\text {in }} \approx \frac{\Delta R}{2 R} V_{\text {in }}
\end{array}\right\}
$$

Thus the fully active bridge configuration has twice the sensitivity of the half active bridges to pressure changes and are made with fewer diaphragms. If the resistor layout of Figure 1 was modified to accommodate two separate radial resistors on one diaphragm (and no circumferential resistors), a single diaphragm, half active sensor could be made. The necessary dummy resistors could be placed close to the diaphragm. The resulting sensor would have half the sensitivity, but occupy less area.

\section{RESULTS AND DISCUSSION}

Several device lots were fabricated and tested in a small pressure chamber constructed of standard stainless steel pipe fittings, shown schematically in Figure 5. A typical response curve is shown in Figure 6. The measurements were performed

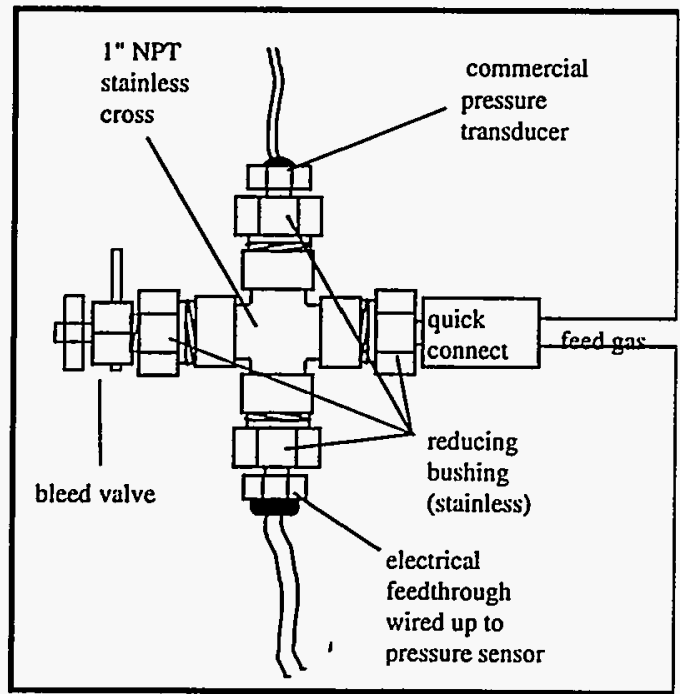

Figure 5. Pressure testing apparatus

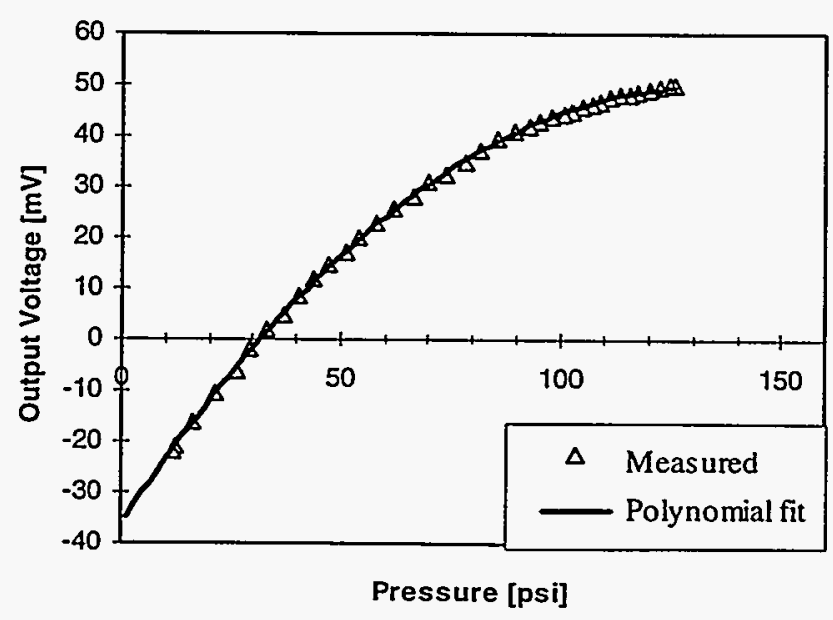

Figure 6. Typical response curve of fully active bridge sensor. Applied voltage is $10 \mathrm{~V}$. 
with compressed air under static conditions using an Omega PX300 commercial pressure sensor with $0.25 \%$ full scale accuracy. The response has good linearity below 40 psia, but begins to saturate above that level. The non-linearity could be due to the diaphragm contacting the substrate, which is consistent with the $\mathrm{P}_{\max }$ of a $0.8 \mu \mathrm{m}$ thick diaphragm shown in Table 1. However, other factors, such as stress stiffening and imperfectly clamped edges are not taken into account by this analysis.

The data in Figure 6 were fit by the least squares method to a second order polynomial of the form:

$$
\frac{V_{\text {out }}}{V_{\text {in }}}=C_{2} P^{2}+C_{1} P+C_{0}
$$

The constants $C_{2}, C_{l}$, and $C_{0}$ represent the nonlinearity, sensitivity, and zero pressure offset voltage of the sensor, respectively. These constants were determined for a number of sensors in all three configurations outlined in section 2.3. The results are shown in the histograms of Figure 7,8 and 9. Since Equation (5) is normalized by applied voltage, the units of volts appear in the denominator of all constants. The insets of the offset voltage graphs are histograms of the data points near zero offset with finer data bins. The large variation of all constants suggests poor process control during fabrication. Part of this variation is attributed to an improper dopant activation anneal for the piezoresistors. Additionally, the thickness control and sidewall etching issues mentioned earlier may contribute to variance. Non-functional sensors were optically examined to identify the source of failure. Of the samples that were examined, all had cracked or broken diaphragms. Principal causes of diaphragm damage are improper handling and hydrostatic pressures during wet cleans after the diaphragms have been sealed.

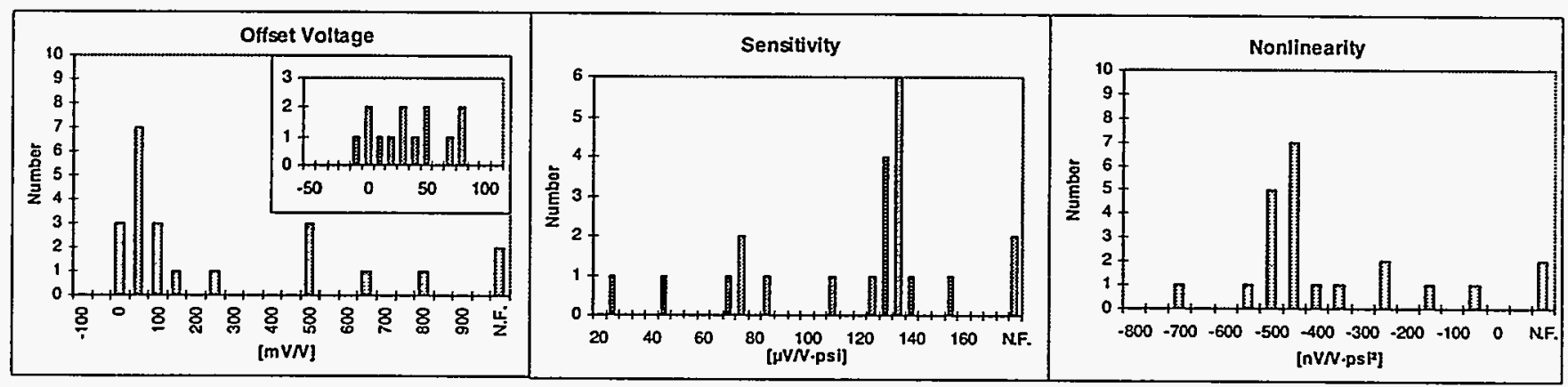

Figure 7. Histograms for full bridge sensors. Applied voltage is $10 \mathrm{~V}$, and there were 22 samples total. N.F.=non functional.

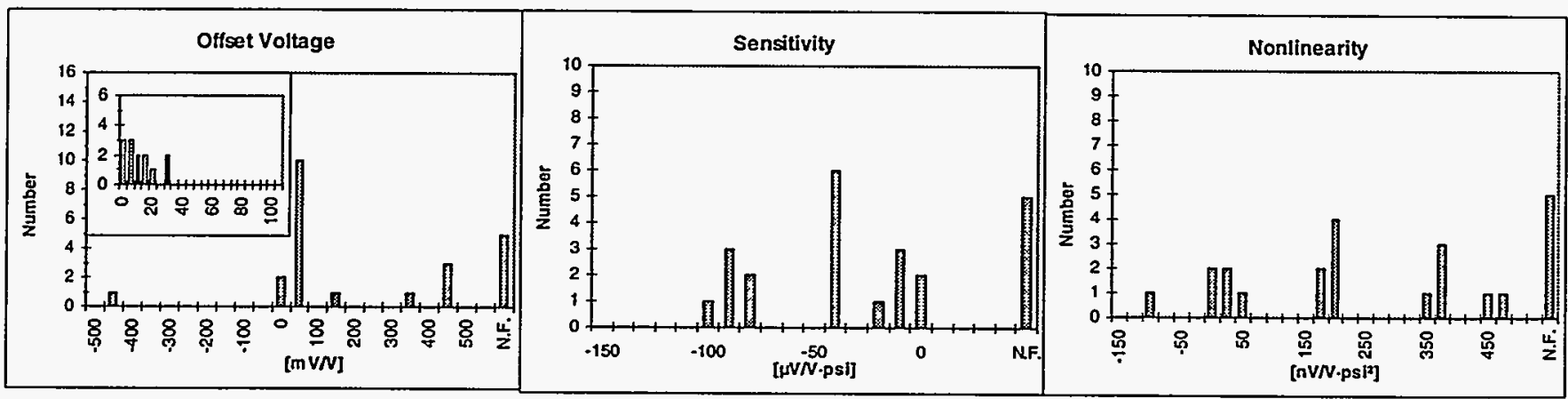

Figure 8. Histograms for half active circumferential bridge sensors. Applied voltage is $10 \mathrm{~V}$, and there were 23 samples total. N.F.=non functional.

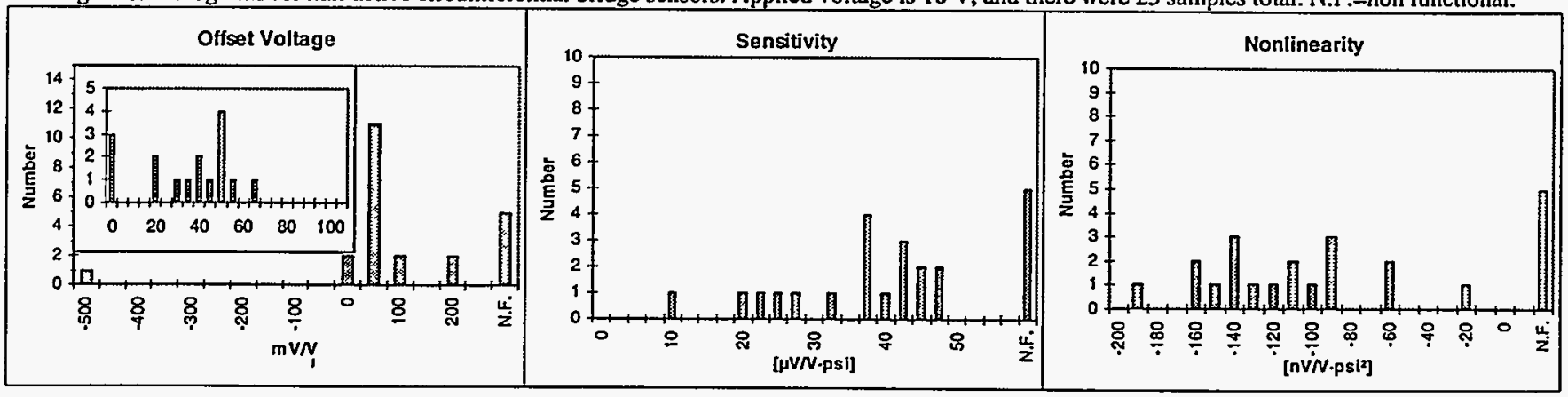

Figure 9. Histograms for half active radial bridge sensors. Applied voltage is $10 \mathrm{~V}$, and there were 23 samples total. N.F.=non functional. 
Some of the polysilicon piezoresistors were examined for noise characteristics. Preliminary results of the noise power in Figure 10 suggest that $1 / f$ noise dominates.

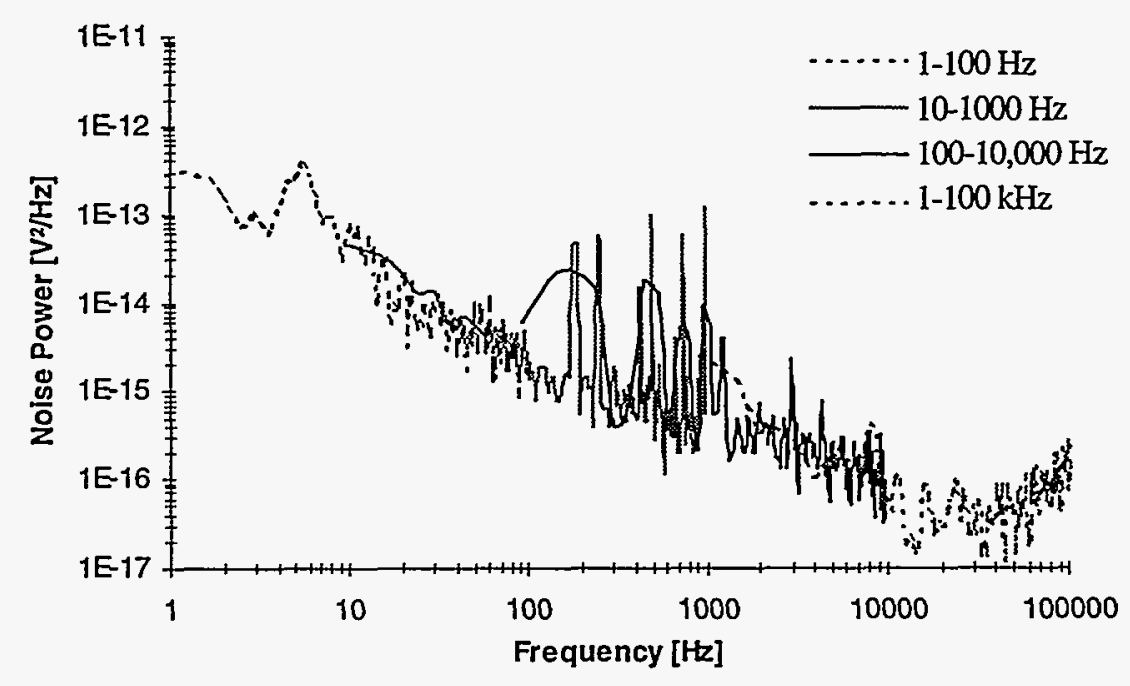

Figure 10. Noise power for a single polysilicon piezoresistor measured over several decades of frequency ranges. Each trace corresponds to the two decade bandwidth indicated.

\section{CONCLUSION}

A pressure sensor array has been designed and fabricated. The sensors are based on silicon nitride diaphragms with polysilicon piezoresistors. All tools and materials were CMOS compatible. Some design considerations regarding diaphragm geometry and Wheatstone bridge configuration were presented and discussed Several processing issues were addressed, including diaphragm patterning, release etching, and aluminum step coverage. Offset voltage, sensitivity, and nonlinearity of several sensors were extracted from least squares fits of measured data. Preliminary data indicate that the dominant source of noise is $1 / f$ noise.

\section{ACKNOWLEDGMENTS}

This work was performed and supported at Sandia National Laboratories by the U.S. Department of Energy under contract DE-AC04-94AL85000

Special thanks to Jeff Sniegowski, who provided design advice, and to the staff and operators of Sandia National Laboratories' Microelectronics Development Laboratory, without whom the devices could not have been made.

\section{REFERENCES}

1 J.H. Smith, S. Montague, J.J. Sniegowski, and P.J. McWhorter, "Embedded micromechanical devices for the monolithic integration of MEMS with CMOS," submitted to the International Electron Device Meeting, IEDM '95, sponsored by IEEE, Washington D.C. December, 1995.

2 B. Kloeck and N.F. de Rooij, "Mechanical sensors," in Semiconductor Sensors, ed. S.M. Sze, John Wiley \& Sons, Inc. pp. 153-204 (1994).

3 D.W. Burns, "Micromechanics of integrated sensor and the planar processed pressure transducer," Ph.D. Thesis, Department of Materials Science, University of Wisconsin at Madison, 1988. 
(1987)

S. Timoshenko and S. Woinosky-Krieger, Theory of Plates and Shells, McGraw Hill Classic Textbook Reissue,

${ }^{5}$ K.H.-L. Chau, C.D. Fung, P.R. Harris, and J.G. Panagou, “Over-range behavior of sealed-cavity polysilicon pressure sensors", Sensors and Actuators A, 28, pp. 147-152 (1991).

${ }^{6}$ W.P. Eaton and J.H. Smith, "A CMOS-compatible, surface-micromachined pressure sensor for aqueous ultrasonic applications," Proceedings of Smart Materials and Structures 95, SPIE, vol 2448, pp. 258-265 (1995).

${ }^{7}$ L.H. Chang, "A study of PSG sacrificial etch solutions used in surface micromachining processing," Proceedings of the Symposium on Microstructures and Microfabricated Systems, the Electrochemical Society, vol 94-14, pp. 79-85.

${ }^{8}$ L. Lin, K.M. McNair, R.T. Howe, and A.P. Pisano, "Vacuum-encapsulated lateral microresonators," Proceedings of the 7th International Conference on Solid-State Sensors and Actuators, Transducers 93, IEEE Press, pp. 270-273 (1993).

9 P.J. French and A.G.R. Evans, "Piezoresistance in polysilicon and its applications to strain gauges", Solid State Electronics, 32(1), pp. 1-10 (1989).

${ }^{10}$ H. Guckel, "Surface micromachined pressure transducers," Sensors and Actuators A, A28, pp. 133-146 (1991).

${ }^{11}$ K.F. Dryden, "The constant current loop: a new paradigm for resistance signal conditioning," NASA Technical Memorandum 104260 (1992).

12 K.F. Anderson, Sensors, April 1994, pp.34-58.

\section{DISCLAIMER}

This report was prepared as an account of work sponsored by an agency of the United States perther the United States Government nor any agency thereof, nor any of their Government. Neither the United States Governied, or assumes any legal liability or responsiemployees, makes any warranty, express or implied, or assumes any lion, apparatus, product, or bility for the accuracy, completeness, or usefulness of any ininge privately owned rights. Referprocess disclosed, or represents that its use would not infringe privately owe name, trademark, ence herein to any specific commercial product, process, or se imply its endorsement, recommanufacturer, or otherwise does not necessarily constite or any agency thereof. The views mendation, or favoring by the United States Governmessarily state or reflect those of the

and opinions of authors expressed herein do not 